01

\title{
Моделирование характеристического излучения сильно ионизованной лазерной плазмы
}

\author{
(C) М.В. Седов ${ }^{1}$, К.Ю. Платонов ${ }^{2}$, А.А. Андреев ${ }^{1}$ \\ ${ }^{1}$ Санкт-Петербургский государственный университет, \\ 199034 Санкт-Петербург, Россия \\ ${ }^{2}$ Санкт-Петербургский политехнический университет Петра Великого, \\ 195251 Санкт-Петербург, Россия \\ e-mail: m.sedov@spbu.ru
}

Поступила в редакцию 29.06.2018 г.

\begin{abstract}
Представлена аналитическая модель, которая рассчитывает рекомбинационное излучение в Не и Ly характеристические линии плазмы, образованной в результате облучения твердой мишени интенсивным лазерным импульсом пикосекундной длительности Для калибровки данная модель сравнивалась с компьютерным кодом FLYCHK. По итогам сравнения область применимости модели лежит в интервале температур основного объема плазмы $200-2000 \mathrm{eV}$ и электронной плотности $10^{23}-10^{24} \mathrm{~cm}^{-3}$. Преимуществом данной аналитической модели является простота, что позволяет использовать ее для быстрых оценок светимости характеристических линий плазмы при заданной температуре и плотности, или наоборот, определять плотность и температуру плазменного источника по относительной высоте и структуре излучаемых линий.
\end{abstract}

DOI: $10.21883 / O S .2018 .12 .46931 .183-18$

\section{Введение}

Лазерно-плазменные источники рентгеновского излучения получают все большее распространение. Их малый размер, высокая пространственная когерентность источника, квазимонохроматичность и сверхкороткая длительность рентгеновского высвета позволяют эффективно использовать лазерную плазму, например, для рентгеновской микроскопии с высоким разрешением, радиографии, фотовозбуждения ядерных реакций и других приложений.

В последние несколько десятилетий были проведены экспериментальные исследования и моделирование $K \alpha$-излучения лазерной плазмы при взаимодействии сверхкоротких лазерных импульсов и различных мишеней [1-11]. Используя различные элементы в качестве материала мишеней, можно получить $K \alpha$-высвет с энергией фотона в диапазоне $1-100 \mathrm{keV}$ [2]. Большое количество работ было посвящено оптимизации коэффициента конверсии лазерного излучения в $K \alpha$-линию: изменение интенсивности лазера, масштаба неоднородности плазмы, размеров и формы мишени [6-9]. Максимальный достигнутый экспериментально коэффициент конверсии в $K \alpha$-линию при длительности импульса менее 1 ps $\sim 10^{-4}[2,3,8]$. Помимо $K \alpha$-излучения, линии рекомбинационного излучения горячей лазерной плазмы (такие как $\mathrm{Ly}-\alpha$ - и $\mathrm{He}-\alpha$-линии) также являются одним из возможных источников ультракороткого рентгеновского излучения. В настоящее время максимальный полученный коэффициент конверсии лазерного излучения в Не- $\alpha$-линию составляет $\sim 10^{-3}[12,13]$ при лазерной интенсивности $10^{17} \mathrm{~W} / \mathrm{cm}^{2}$, что превышает конверсию в К $\alpha$-линию.
С другой стороны, спектр излучения лазерной плазмы можно использовать для диагностики ее текущего состояния. Измерения градиента электронной температуры и плотности помогают в изучении транспорта энергии в релятивистской лазерной плазме твердотельной плотности. Стандартным методом определения плотности и температуры в плазме является рентгеновская спектроскопия $[14,15]$. Плотная и горячая плазменная среда вызывает смещение и уширение спектральных линий. Сравнение измеренных ширин спектральных линий, их относительных интенсивностей с результатами расчетов позволяет определить параметры плазмы [16,17]. Рекомбинационные Не- и Lу-линии в этом ключе особенно интересны. Эти линии легко детектировать, в то же время их относительная высота чувствительна к электронной температуре, а их ширина и тонкая структура чувствительны к плазменной плотности. Это делает их привлекательными для использования в рентгеновской спектроскопии. Стандартный способ определения параметров плазмы - построение синтетического спектра, максимально соответствующего экспериментально измеренному с помощью различных моделирующих программ. В настоящее время создано большое количество подобных кодов - как коммерческих, так и распространяемых по свободной лицензии [18-20]. Однако они все сложны в освоении, а проведение аккуратного моделирования и обработка результатов занимают много времени.

\section{Описание модели}

В данной работе представлена аналитическая модель, рассчитывающая излучение плазмы в линии He и Ly для 
заданной температуры и плотности плазмы. Она базируется на разработанной ранее модели зависимости излучения плазмы в Не- $\alpha$-линию от времени задержки и интенсивности лазерного предымпульса [12]. Модель была доработана и расширена расчетом излучения Ly- $\alpha$ линии. Для простоты рассмотрим сначала излучение в Не- $\alpha$-линию. В приближении, что число ионов зависит только от ионизации и рекомбинации, для Не-подобных ионов можно записать

$$
\partial N_{1} / \partial t=n_{e} N_{i 0}\left\langle\sigma v_{e}\right\rangle_{\mathrm{He}}-N_{1} \gamma_{0},
$$

$\langle\sigma v e\rangle_{\mathrm{He}}-$ коэффициент ионизации для Li-подобного иона, $\gamma_{0}$ - коэффициент рекомбинации. Для населенности $N_{2}$ верхнего уровня скоростное уравнение запишется следующим образом:

$$
\partial N_{2} / \partial t=-A_{21} N_{2}-\langle\sigma v e\rangle_{21} n_{e} N_{2}+\left\langle\sigma v_{e}\right\rangle_{12} N_{1} n_{e},
$$

где $A_{21}$ - вероятность радиационного перехода (обратное время жизни) для $\mathrm{He}-\alpha$-линии, $\left\langle\sigma v_{e}\right\rangle_{12}=$ $\left.=<\sigma v_{e}\right\rangle_{21}\left(g_{2} / g_{1}\right) \exp \left(-E_{21 \mathrm{He}} / T_{e}\right)-$ коэффициенты возбуждения и релаксации, $g_{1}=1$ и $g_{2}=3$ - статистические веса. Исходные населенности вычисляются в предположении, что все ионы находятся в нижнем состоянии: $N_{1}=n_{i}$. Тогда интенсивность линии излучения $2 \rightarrow 1$-перехода

$$
\partial W_{21} / \partial t=E_{21} A_{21} N_{2}(t)
$$

Чтобы найти интенсивность Не- $\alpha$-линии, нужно решить систему уравнений (1)-(3). Эту модель можно легко расширить на расчет Ly- $\alpha$-линии, учтя, что часть Не-подобных ионов ионизируется до Н-подобных, а часть их, в свою очередь, теряет последний электрон и становится полностью ионизованной. Тогда уравнение (1) переходит в систему уравнений

$$
\begin{gathered}
\partial N_{1 \mathrm{He}} / \partial t=n_{e}\left(N_{1 \mathrm{Li}}(t)-N_{1 \mathrm{He}}(t)\right)\left\langle\sigma v_{e}\right\rangle_{\mathrm{Li}} \\
-\gamma_{1}(t) N_{1 \mathrm{He}}(t)-\partial N_{1 \mathrm{H}} / \partial t \\
\partial N_{1 \mathrm{H}} / \partial t=n_{e}\left(N_{1 \mathrm{He}}(t)-N_{1 \mathrm{H}}(t)\right)\left\langle\sigma v_{e}\right\rangle_{\mathrm{He}} \\
-\gamma_{2}(t) N_{1 \mathrm{H}}-\partial N_{1 \alpha} / \partial t \\
\partial N_{1 \alpha} / \partial t=n_{e}\left(N_{1 \mathrm{H}}(t)-N_{1 \alpha}(t)\right)\left\langle\sigma v_{e}\right\rangle_{\mathrm{H}}-\gamma_{3}(t) N_{1 \alpha}(t) .
\end{gathered}
$$

Здесь $\gamma_{1,2,3}(t)=4 \cdot 10^{-27} C_{1,2,3} Z^{3} T_{e}(t)^{-9 / 2} n_{e}(t)^{2}\left[\mathrm{~s}^{-1}\right]$ коэффициенты рекомбинации в приближении диффузной модели [21]. $C_{1}, C_{2}, C_{3}$ - численные константы $(\sim 1)$, $n_{e}$ - электронная плотность $\left[\mathrm{cm}^{-3}\right]$, температура $T_{e}<J_{Z}-$ потенциала ионизации, $N_{\mathrm{Li}}-$ число $\mathrm{Li}$-подобных ионов, $N_{1 \mathrm{He}}$ и $N_{1 \mathrm{H}}-$ это число Неи Н-подобных ионов, $\left\langle\sigma v_{e}\right\rangle_{\mathrm{Li}},\left\langle\sigma v_{e}\right\rangle_{\mathrm{He}}$ и $\left\langle\sigma v_{e}\right\rangle_{\mathrm{H}}-$ потенциалы ионизации для Li-, He- и Н-подобных ионов, для их определения используется численное выражение из [22], которое можно аппроксимировать как $\left\langle\sigma v_{e}\right\rangle_{i} \approx \sigma_{0}\left(T_{e}\right)^{0.5} \mathrm{Ji}^{-2} \exp \left(-J_{i} / T_{e}\right)$, $\sigma_{0}=2 \cdot 10^{-12} \mathrm{~cm}^{2} / \mathrm{keV}^{3 / 2}$.
Характерная скорость ионизации Li-подобного иона порядка $10^{-11} \mathrm{~cm}^{3} / \mathrm{s}$. Поэтому за время порядка $170 \mathrm{fs}$. устанавливается стационарное решение

$$
\begin{gathered}
N_{1 \mathrm{He}}(t)=N_{1 \mathrm{Li}}(t) \frac{n_{e}(t)\left\langle\sigma v_{e}\right\rangle_{\mathrm{Li}}}{\left.n_{e}(t)\left\langle\sigma v_{e}\right\rangle_{\mathrm{Li}}+\gamma_{1}(t)\right)}-N_{1 \mathrm{H}}(t), \\
N_{1 \mathrm{H}}(t)=N_{1 \mathrm{He}}(t) \frac{n_{e}(t)\left\langle\sigma v_{e}\right\rangle_{\mathrm{He}}}{\left.n_{e}(t)\left\langle\sigma v_{e}\right\rangle_{\mathrm{He}}+\gamma_{2}(t)\right)}-N_{1 \alpha}(t), \\
N_{1 \alpha}(t)=N_{1 \mathrm{H}}(t) \frac{n_{e}(t)\left\langle\sigma v_{e}\right\rangle_{\mathrm{H}}}{\left.n_{e}(t)\left\langle\sigma v_{e}\right\rangle_{\mathrm{H}}+\gamma_{3}(t)\right)} .
\end{gathered}
$$

Уравнение (4) также имеет общее решение $\left(\left.N_{1 \mathrm{He}}\right|_{t=0}=0,\left.\quad N_{1 \mathrm{H}}\right|_{t=0}=0\right), \quad$ которое переходит $\quad$ в стационарное на больших временах:

$$
\begin{aligned}
& N_{1 \mathrm{He}}(t)=\int_{0}^{t_{1}} d t_{1} N_{1 \mathrm{Li}}\left(t_{1}\right)\left\langle\sigma v_{e}\left(t_{1}\right)\right\rangle_{\mathrm{Li}} \\
& \times \exp \left(\int_{t_{1}}^{t} d t_{2}\left(n_{e}\left(t_{2}\right)\left\langle\sigma v_{e}\left(t_{1}\right)\right\rangle_{\mathrm{Li}}+\gamma_{2}\left(t_{2}\right)\right)\right)-N_{1 \mathrm{H}}(t), \\
& N_{1 \mathrm{H}}(t)=N_{1 \mathrm{He}} \int_{0}^{t_{1}} d t_{1} n_{e}\left(t_{1}\right)\left\langle\sigma v_{e}\left(t_{1}\right)\right\rangle_{\mathrm{He}} \\
& \times \exp \left(\int_{t_{1}}^{t} d t_{2}\left(n_{e}\left(t_{2}\right)\left\langle\sigma v_{e}\left(t_{1}\right)\right\rangle_{\mathrm{He}}+\gamma_{2}\left(t_{2}\right)\right)\right)-N_{1 \alpha}(t), \\
& N_{1 \alpha}(t)=N_{1 \mathrm{H}} \int_{0}^{t_{1}} d t_{1} n_{e}\left(t_{1}\right)\left\langle\sigma v_{e}\left(t_{1}\right)\right\rangle_{\mathrm{H}} \\
& \times \exp \left(\int_{t_{1}}^{t} d t_{2}\left(n_{e}\left(t_{2}\right)\left\langle\sigma v_{e}\left(t_{1}\right)\right\rangle_{\mathrm{H}}+\gamma_{2}\left(t_{2}\right)\right)\right) .
\end{aligned}
$$

Для возбужденного уровня $N_{2}$ скоростные уравнения записываются следующим образом:

$$
\begin{aligned}
\partial N_{2 \mathrm{He}} / \partial t & =-A_{21 \mathrm{He}} N_{2 \mathrm{He}}-\left\langle\sigma v_{e}\right\rangle_{21 \mathrm{He}} N_{2 \mathrm{He}}(t) \\
& +\left\langle\sigma v_{e}\right\rangle_{12 \mathrm{He}} N_{1 \mathrm{He}}(t) \\
\partial N_{2 \mathrm{H}} / \partial t & =-A_{21 \mathrm{H}} N_{2 \mathrm{H}}-\left\langle\sigma v_{e}\right\rangle_{21 \mathrm{H}} N_{2 \mathrm{H}}(t) \\
& +\left\langle\sigma v_{e}\right\rangle_{12 \mathrm{H}} N_{1 \mathrm{H}}(t) .
\end{aligned}
$$

Здесь $A_{21}-$ коэффициент спонтанной эмиссии для $\mathrm{He}-\alpha$ - и $\mathrm{Ly}-\alpha$-линий (например, для алюминия $\left.A_{21 \mathrm{He}}=2.7 \cdot 10^{13}, A_{21 \mathrm{H}}=1.36 \cdot 10^{13} \mathrm{~s}^{-1}\right)$,

$$
\begin{gathered}
\left\langle\sigma v_{e}\right\rangle_{12 \mathrm{He}}=\left\langle\sigma v_{e}\right\rangle_{21 \mathrm{He}}\left(g_{2} / g_{1}\right) \exp \left(-E_{21 \mathrm{He}} / T_{e}(t)\right), \\
\left\langle\sigma v_{e}\right\rangle_{12 \mathrm{H}}=\left\langle\sigma v_{e}\right\rangle_{21 \mathrm{H}}\left(g_{2} / g_{1}\right) \exp \left(-E_{21 \mathrm{He}} / T_{e}(t)\right), \\
\left\langle\sigma v_{e}\right\rangle_{12 \mathrm{He}} \approx\left\langle\sigma v_{e}\right\rangle_{12 \mathrm{H}}=10^{-9} \mathrm{~cm}^{3} / \mathrm{s} \\
\left(T_{e} \sim 300-700 \mathrm{eV}\right)[23] .
\end{gathered}
$$



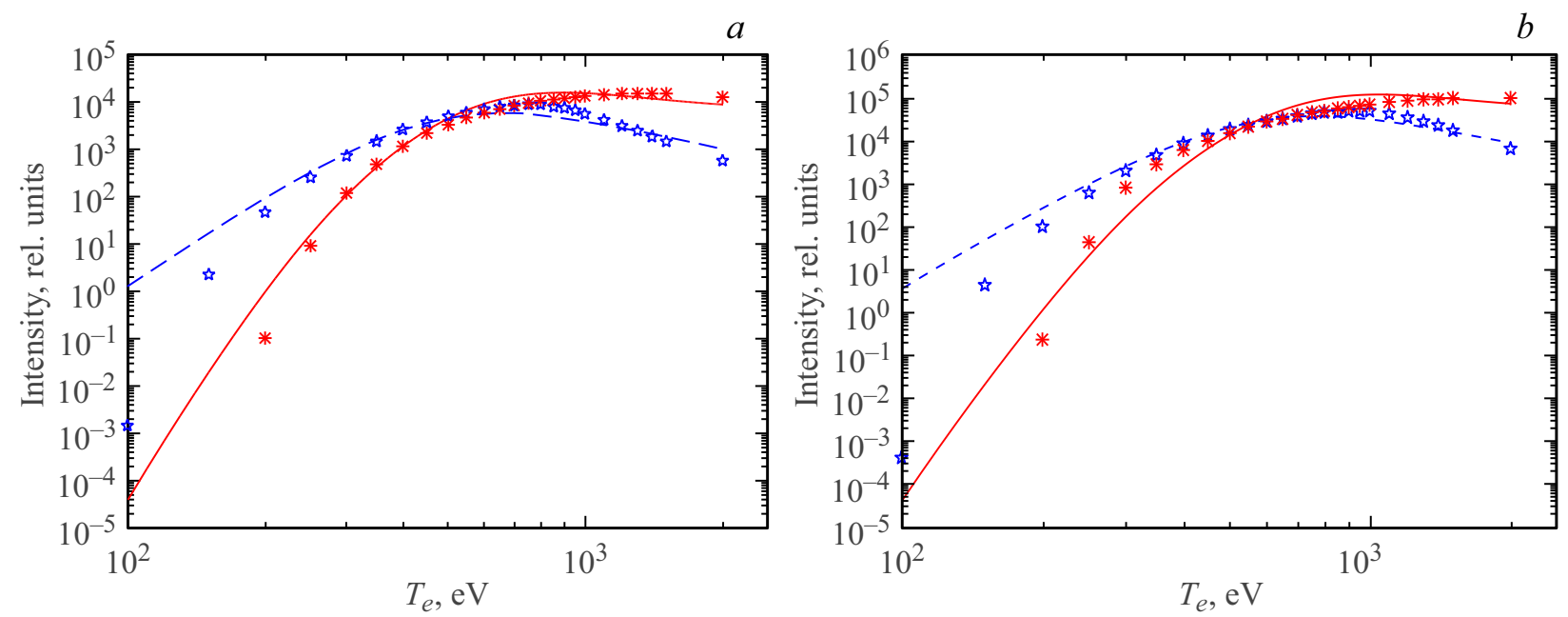

Рис. 1. Зависимость интенсивности Не- $\alpha$ - (штриховая линия и пятиконечные звезды) и Ly- $\alpha$ - (сплошная линия и звездочки) линий от температуры. Электронная плотность постоянна и равна $(a) 1 \cdot 10^{23} \mathrm{~cm}^{-3},(b) 8 \cdot 10^{23} \mathrm{~cm}^{-3}$. Сплошные линии - расчет по аналитической модели, точки - FLYCHK-расчет.

Нестационарное решение:

$$
\begin{aligned}
& \left.N_{2 \mathrm{He}}(t)=\int_{0}^{t_{1}} d t_{1} N_{1 \mathrm{He}}\left(t_{1}\right) \sigma v_{e}\left(t_{1}\right)\right\rangle_{12 \mathrm{He}} \\
& \times \exp \left(-\int_{t_{1}}^{t} d t_{2}\left(N_{1 \mathrm{He}}\left(t_{1}\right)\left\langle\sigma v_{e}\left(t_{1}\right)\right\rangle_{21 \mathrm{He}}+A_{21 \mathrm{He}}\right)\right), \\
& \left.N_{2 \mathrm{H}}(t)=\int_{0}^{t_{1}} d t_{1} N_{1 \mathrm{H}}\left(t_{1}\right) \sigma v_{e}\left(t_{1}\right)\right\rangle_{12 \mathrm{H}} \\
& \times \exp \left(-\int_{t_{1}}^{t} d t_{2}\left(N_{1 \mathrm{H}}\left(t_{1}\right)\left\langle\sigma v_{e}\left(t_{1}\right)\right\rangle_{21 \mathrm{H}}+A_{21 \mathrm{H}}\right)\right) .
\end{aligned}
$$

Стационарное решение:

$$
\begin{aligned}
& N_{2 \mathrm{He}}(t)=N_{1 \mathrm{He}} \frac{n_{e}(t)\left\langle\sigma v_{e}\right\rangle_{12 \mathrm{H}}}{n_{e}(t)\left\langle\sigma v_{e}\right\rangle_{21 \mathrm{H}}+A_{21 \mathrm{H}}} \\
& \approx N_{1 \mathrm{He}}(t) \frac{g_{2}}{g_{1}} \exp \left(-\frac{E_{21 \mathrm{He}}}{T_{e}(t)}\right), \quad g_{2}=3, \quad g_{1}=1, \\
& N_{2 \mathrm{H}}(t)=N_{1 \mathrm{H}} \frac{n_{e}(t)\left\langle\sigma v_{e}\right\rangle_{12 \mathrm{H}}}{n_{e}(t)\left\langle\sigma v_{e}\right\rangle_{21 \mathrm{H}}+A_{21 \mathrm{H}}} \\
& \approx N_{1 \mathrm{H}}(t) \frac{g_{2}}{g_{1}} \exp \left(-\frac{E_{21 \mathrm{H}}}{T_{e}(t)}\right) .
\end{aligned}
$$

Стационарное решение устанавливается за время, меньшее, чем $A_{21 \mathrm{H}}^{-1}$ (для $\mathrm{Al} A_{21 \mathrm{H}}^{-1} \approx 56 \mathrm{fs}$ ).

Интенсивность излучения на $2 \rightarrow 1$-переходе для $\mathrm{He}-\alpha$ - и $\mathrm{Ly}-\alpha$-линий находится из уравнений

$$
\begin{gathered}
\partial W_{21 \mathrm{He}} / \partial t=E_{21 \mathrm{He}} A_{21 \mathrm{He}} N_{2 \mathrm{He}}(t), \\
\partial W_{21 \mathrm{H}} / \partial t=E_{21 \mathrm{H}} A_{21 \mathrm{H}} N_{2 \mathrm{H}}(t)
\end{gathered}
$$

(для алюминия $E_{21 \mathrm{He}}=1869 \mathrm{eV}, E_{21 \mathrm{H}}=2010 \mathrm{eV}$ ). Интегрируя по времени, получаем

$$
\begin{gathered}
W_{21 \mathrm{He}}(t)=E_{21 \mathrm{He}} A_{21 \mathrm{He}} \int_{0}^{t} N_{2 \mathrm{He}}\left(t_{1}\right) d t_{1}, \\
W_{21 \mathrm{H}}(t)=E_{21 \mathrm{H}} A_{21 \mathrm{H}} \int_{0}^{t} N_{2 \mathrm{H}}\left(t_{1}\right) d t_{1} .
\end{gathered}
$$

Таким образом, мы получаем систему уравнений (5)-(7), которую можно решить численно.

Модель имеет 3 подгоночных константы $C_{1}, C_{2}, C_{3}$. Температура электронов и количество Li-подобных ионов задаются в качестве начальных параметров.

\section{Калибровка и верификация модели}

Для калибровки модели было проведено сравнение с программой FLYCHK [18]. Этот код использует гидродинамическую модель плазмы с учетом столкновений и излучения. FLYCHK моделирует поведение плазмы в одной точке (нулевая размерность), но также может учитывать поглощение излучения в плазме (параметр opacity), и в этом процессе плазма рассматривается как одномерный жгут с одинаковой плотностью и температурой на всем его протяжении. При калибровке мы исходили из предположения, что температура плазмы достаточна для того, чтобы перевести все ионы как минимум в Li-подобное состояние, т. е. $N_{1 \mathrm{Li}}=N_{i}=Z n_{e}$. При выборе констант мы ориентировались на максимальное сходство с FLYCHK-моделированием в диапазоне температур $300-600 \mathrm{eV}$. Как видно из рис. 1, результаты, полученные из модели, хорошо совпадают с численными расчетами в интервале температур 


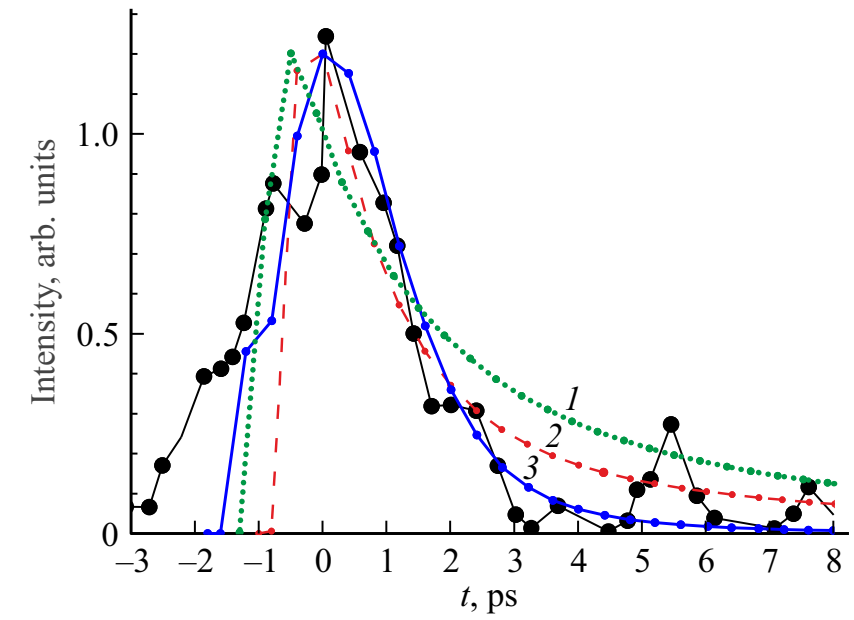

Рис. 2. Временная зависимость интенсивности Не- $\alpha$-линии: экспериментально измеренная [24] (черные точки), $1-$ полученная с помощью расчетов по аналитической модели и разбиением лазерного пятна на 3 части с разными инттенсивностями. 2 - расчет по аналитической модели с одной интенсивностью $5 \cdot 10^{17} \mathrm{~W} / \mathrm{cm}^{2} .3-$ расчет с помощью численного кода FLYCHK $\left(5 \cdot 10^{17} \mathrm{~W} / \mathrm{cm}^{2}\right)$.

$200-2000 \mathrm{eV}$ и электронной плотности $1-8 \cdot 10^{23} \mathrm{~cm}^{-3}$ при $C_{1}=150, C_{2}=0.25, C_{3}=0.3$.

Рассмотрим расчет временной зависимости излучения плазмы в Не- $\alpha$-линию в сравнении с экспериментальными результатами из [24]. В этой экспериментальной работе использовалась лазерная экспериментальная установка ATLAS с энергией $250 \mathrm{~mJ}$ и длительностью импульса 150 fs. Лазерное излучение фокусировалось на мишень в пятно радиусом $10 \mu \mathrm{m}$, с пиковой интенсивностью $\approx 5 \cdot 10^{17}$ и средней интенсивностью в пятне $\sim 1 \cdot 10^{17} \mathrm{~W} / \mathrm{cm}^{2}$. В качестве мишени использовались полированные алюминиевые диски. Рекомбинационные спектры записывались на стрик-камеру с временным разрешением $1.7 \mathrm{ps}$. Измеренное лазерное поглощение составило $\approx 40 \%$.

Для моделирования взаимодействия лазерного излучения с алюминиевой мишенью использовалась одномерная гидродинамическая программа MEDUSA [25]. Она была разработана в Лаборатории Резерфорда-Эплтона для исследования некоторых гидродинамических и плазменных процессов, происходящих в капсуле с топливом для термоядерной реакции, которая облучалась лазерным излучением. Плазма рассматривается с точки зрения одножидкостной двухтемпературной модели. Лазерное излучение рассматривается как внешний источник тепла, который поглощается в ячейке с критической плазменной плотностью.

Вообще говоря, если интенсивность лазерного излучения превышает $10^{16} \mathrm{~W} / \mathrm{cm}^{2}$, гидродинамическая модель приближается к своему пределу применимости, поскольку горячие электроны начинают обгонять тепловую волну, образующуюся в точке поглощения лазерного излучения. Это вызывает нелокальный перенос тепла, который не описывается гидродинамической моделью. B MEDUSA это учитывается следующим образом: задается процент поглощенной лазерной энергии, перешедшей в энергию горячих электронов. Затем они распространяются вглубь мишени с постоянной скоростью. Когда эти электроны достигают другого края мишени, то их энергия равномерно распределяется по всем ячейкам, что отражает нагрев мишени быстрыми электронами до прихода тепловой волны. Также отметим, что основной вклад в излучение вносит горячая плазма в окрестности точки с критической плотностью, которая после окончания воздействия лазерного импульса быстро ( 100 fs) приходит к максвелловскому состоянию и хорошо описывается гидродинамической моделью. Поэтому мы считаем, что для оценки временной эволюции приповерхностной плазмы программу MEDUSA можно использовать вплоть до лазерной интенсивности $\sim 10^{18} \mathrm{~W} / \mathrm{cm}^{2}$, когда уже нельзя пренебрегать релятивистскими эффектами.

Численное моделирование проводилось двумя способами: со средней интенсивностью в пятне и с разбиением лазерного пятна на 3 части - круг с радиусом в $3 \mu \mathrm{m}$ и интенсивностью $4 \cdot 10^{17} \mathrm{~W} / \mathrm{cm}^{2}$ и 2 кольца от 3 до 6 и от 6 до $10 \mu \mathrm{m}$ с интенсивностями $2 \cdot 10^{17}$ и $7 \cdot 10^{16} \mathrm{~W} / \mathrm{cm}^{2}$ соответственно. Остальные параметры гидродинамического моделирования были следующими: толщина мишени $10 \mu \mathrm{m}$, равномерное разбиение на 250 ячеек, полное лазерное поглощение составляло $40 \%$, конверсия в горячие электроны $10 \%$ от поглощенной энергии. Температура и плотность плазмы выводилась для всех ячеек с интервалом в $200 \mathrm{fs}$. Затем эти результаты обрабатывались скриптом, написанным в среде MATLAB - для каждой ячейки считался выход в Не- $\alpha$-линию по аналитической модели, и результаты

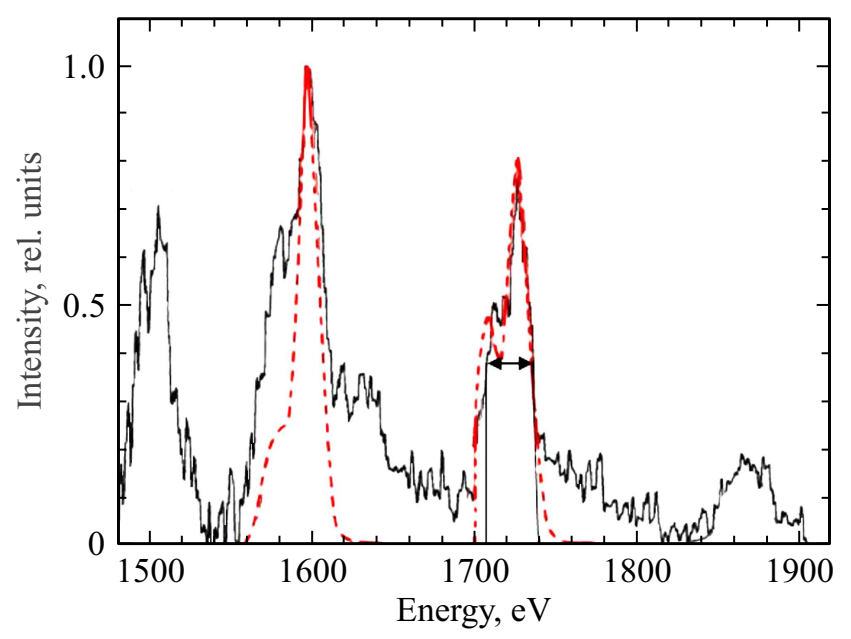

Рис. 3. Экспериментальный спектр излучения плазмы из работы [17] (сплошная линия) и его аппроксимация FLYмоделированием (штриховая линия) при следующих параметpax: бесконечный столб плазмы с температурой $550 \mathrm{eV}$ и плотностью $1 \mathrm{~g} / \mathrm{cm}^{3}$. 
суммировались. Для дополнительного сравнения результаты вычислений были также обработаны в FLYCHK. Для расчета с помощью этого кода необходимо проводить усреднение по ячейкам, так как FLYCHK имеет онлайн интерфейс, и временную зависимость излучения необходимо моделировать отдельно для каждой ячейки. Для этого скрипт разбивал выбранную из результатов гидродинамического моделирования область на макроячейки (в данном случае использовалось 10 макроячеек), и для них записывалась временная эволюция электронной плотности, средняя электронная и ионная температуры, а также размер макроячейки с заданным временным шагом (в данном случае $100 \mathrm{fs}$ ), затем каждый из этих файлов запускался в FLYCHK, и результаты суммировались.

Сравнение моделирования с экспериментальными результатами приведено на рис. 2. Так как экспериментальные результаты [24] приведены в относительных единицах, то мы можем сравнивать только скорость затухания $\mathrm{He}-\alpha$-линии со временем. Также отметим, что странные пики на временах 5 и 7 ps считаются авторами работы [24] погрешностью стрик-камеры, реальное излучение Не- $\alpha$-линии заканчивается на 3 ps по шкале на рис. 2.

Как видно из рис. 2, рассчитанная с помощью FLYCHК-кода временная зависимость хорошо описывает экспериментально измеренную. Аналитическая модель описывает эксперимент чуть хуже. Если определять длительность Не- $\alpha$-излучения как ширину на половине интенсивности, то мы получим $\approx 2.7$ ps для эксперимента, 2.5 ps для FLYCHK, $\approx 2$ ps для аналитической модели с одной интенсивностью и $\approx 2.8$ ps для разбиения пятна на 3 части. Отметим также, что скрипту, обрабатывающему результаты расчетов в MEDUSA с помощью модели для работы необходимо порядка минуты, а для FLYCHKрасчетов с 10 ячейками необходимо порядка часа работы - последовательно загружать данные на сервер FLYCHK, запускать моделирование, которое длится примерно $30 \mathrm{~s}$, а затем ждать 5-10 min, пока выгрузятся результаты. Таким образом, расчет по модели проходит примерно в 50 раз быстрее.

\section{Применение аналитической модели для определения параметров лазерной плазмы по ее спектру}

Рассмотрим анализ экспериментального спектра из [17]. В этой работе рассматривалось взаимодействие лазерного излучения длительностью $\sim 1$ ps и интенсивностью $\sim 10^{20} \mathrm{~W} / \mathrm{cm}^{2}$ с алюминиевыми мишенями различной толщины. Спектр излучения вместе с проведенным авторами статьи моделированием в программе FLY приведен на рис. 3.

Из рис. 3 можно оценить ширину Lу-линии примерно в $25 \mathrm{eV}$, а отношение интенсивностей Не- и Lу-линий $W_{\mathrm{He}} / W_{\mathrm{Ly}} \approx 1.3$.

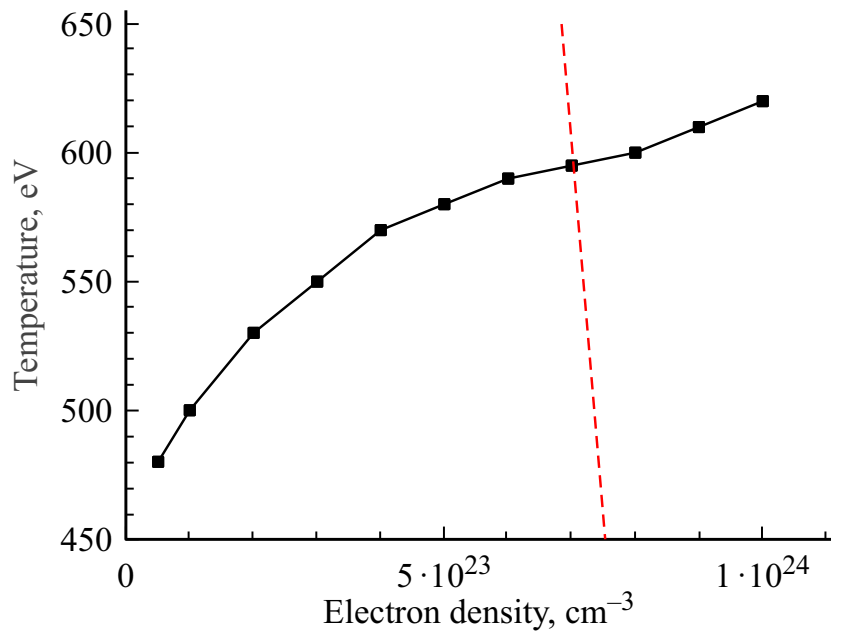

Рис. 4. Зависимость температуры и плотности плазмы, при которых отношение интенсивности Не- и Ly-линий $W_{\mathrm{He}} / W_{\mathrm{Ly}}=1.3$ (черные квадраты). Штриховая линия - плотность плазмы, при которой ширина Ly- $\alpha$-линии $\Delta E=25 \mathrm{eV}$.

Таким образом, зафиксировав отношение интенсивности Не- и Lу-линий можно с помощью аналитической модели по формулам (9)-(11) связать температуру плазмы с еe плотностью. Эта зависимость для $W_{\mathrm{He}} / W_{\mathrm{Ly}}=1.3$ изображена на рис. 4.

Для однозначного определения параметров плазмы необходимо дополнить аналитическую модель оценкой ионной плотности по уширению спектральных линий. Оценим ширину линии $\Delta E$ как сумму допплеровской $\Delta E_{D}$ и столкновительной $\Delta E_{S}$ ширин.

$$
\begin{gathered}
\Delta E_{D}=2 E_{0}\left(\frac{2 k T}{m c^{2}}\right)^{\frac{1}{2}} \sqrt{\ln 2}=1.67 \frac{v_{i}}{c} E_{0}, \quad v_{i}=\sqrt{\frac{2 k T}{m}}, \\
\Delta E_{S}=21.6 \frac{n^{2} R y}{z}\left(N a_{0}^{3}\right)^{\frac{2}{3}} .
\end{gathered}
$$

Здесь $E_{0}-$ энергия спектральной линии, $T$ и $N-$ температура и плотность плазмы, $m$ - масса иона, $n$ - главное квантовое число рассматриваемого уровня, $a_{0}$ - боровский радиус, $R y-$ постоянная Ридберга.

Для оценки столкновительной ширины использована известная формула Хольцмарка водородоподобного иона [26]. Она справедлива в диапазоне плазменной плотности $10^{14}<N<10^{25} \mathrm{~cm}^{-3}$ [15]. Ее размерность зависит от выбора размерности постоянной Ридберга. В данной работе используются электронвольты. Отметим, что допплеровское уширение зависит только от температуры, и в данном диапазоне от 400 до $600 \mathrm{eV}$ ширина линии, рассчитанная по формуле (8), меняется от 0.5 до $0.6 \mathrm{eV}$. Поэтому в данном случае основной вклад вносит столкновительное уширение. По формуле (9) для ширины линии в $25 \mathrm{eV}$ мы получаем плотность плазмы $\approx 7.5 \cdot 10^{23} \mathrm{~cm}^{-3}$. Если учесть и допплеровское уширение, то плотность получается чуть меньше: 
$\approx 7 \cdot 10^{23} \mathrm{~cm}^{-3}$. Кривая параметров, при которых ширина линии составляет $25 \mathrm{eV}$ приведена также на рис. 4.

Таким образом, по точке пересечения двух кривых мы можем определить температуру и плотность плазмы: $N \approx 7 \cdot 10^{23} \mathrm{~cm}^{-3}, T \approx 600 \mathrm{eV}$. В работе [17] наилучшее согласие между экспериментальным и синтетическим спектрами было достигнуто при температуре $550 \mathrm{eV}$ и плотности плазмы $1 \mathrm{~g} / \mathrm{cm}^{3} \approx 5 \cdot 10^{23}$ particle $/ \mathrm{cm}^{3}$. Мы видим, что результаты, полученные с помощью данной аналитической модели достаточно близки к результатам численного расчета: по модели плотность получилась завышенной примерно в полтора раза, а температура завышена примерно на $10 \%$.

\section{Заключение}

В настоящей работе представлена аналитическая модель, рассчитывающая излучение плазмы в Не- и Lyлиниях. Разработанная модель подтверждает идею о том, что для анализа конкретной спектральной линии достаточно учитывать ограниченное число переходов. Это продвигает наше понимание в области лазерной плазменной спектроскопии. Для калибровки, а также определения рабочего диапазона модель была сравнена с серией расчетов в программе FLYCHK. Результаты сравнения показали справедливость модели в диапазоне температур 200-2000 eV для электронной плотности $10^{23}-10^{24} \mathrm{~cm}^{-3}$.

Также было проведено сравнение результатов расчетов с помощью модели с двумя экспериментальными работами: определение длительности высвета в Не- $\alpha$ линию [24] и определение температуры и плотности плазмы по ее спектру [17]. При расчете времени излучения аналитическая модель использовалась в качестве постобработки гидродинамического моделирования. Результаты расчета хорошо совпадают с экспериментальными измерениями и численным моделированием в программе FLYCHK (2.7 ps - измеренное время, 2.8 ps по аналитической модели, 2.5 ps - по FLYCHK-расчету). При определении параметров плазмы по экспериментально измеренному спектру результаты, полученные с помощью данной модели, также удовлетворительно совпали с анализом, выполненным авторами работы [17] с помощью моделирующей программы FLY: отклонения нашей модели составили $\sim 40 \%$ в оценке плотности плазмы и $\sim 10 \%$ в оценке температуры.

Данная модель может использоваться, например, в спектроскопии лазерной плазмы для быстрого определения температуры и плотности плазмы. Знание примерных параметров плазмы, полученных с помощью аналитической модели, существенно облегчает расчет в специализированных моделирующих программax. Еще один возможный вариант применения для постобработки результатов гидродинамического или РIC-моделирования - расчет интенсивности излучения плазмы в Не- и Lу-линиях.

\section{Список литературы}

[1] Salzmann D., Reich Ch., Uschmann I., Forster E., Gibbon P. // Phys. Rev. E. 2002. V. 65. P. 036402.

[2] Ziener Ch., Uschmann I., Stobrawa G., Reich Ch., Gibbon P., Feurer T., Morak A., Dusterer S., Schwoerer H., Forster E., Sauerbrey R. // Phys. Rev. E. 2002. V. 65. P. 066411.

[3] Bastiani S., Rousse A., Geindre J.P., Audebert P., Quoix C., Hamoniaux G., Antonetti A., Gauthier J.C. // Phys. Rev. E. 1997. V. 56. N 6. P. 7179.

[4] Агранат М.Б., Андреев Н.Е., Ащитков С.И., Овчинников А.В., Ситников Д.С. // Письма в ЖЭТФ. 2006. Т. 83. № 2. C. 80-83; Agranat M.B., Andreev N.E., Ashitkov S.I., Ovchinnikov A.V., Sitnikov D.S., Fortov V.E., Shevel'ko A.P. // JETP Letters. 2006. V. 83. N 2. P. 72-74.

[5] Neumayer P., Lee H.J., Offerman D., Shipton E., Kemp A., Kritcher A.L., Doppner T., Back C.A., Glenzer S.H. // High Energy Density Physics. 2009. V. 5. P. 244-248.

[6] Nakano H., Andreev A., Limpouch J. // Appl. Phys. B. 2004. V. 79. P. 469-476. doi 10.1007/s00340-004-1582-7

[7] Khattak F.Y., Percie du Sert O.A.M.B., Riley D., Foster P.S., Divall E.J., Hooker C.J., Langley A.J., Smith J., Gibbon P. // Phys. Rev. E. 2006. V. 74. P. 027401.

[8] Khattak F.Y., Percie du Sert O.A.M.B., Riley D., Edwards M., Mistry P., Tallents G., Foster P.S., Clarke R.J., Hooker E.J., Langley A.J., Neely D., Smith J.M., Spindloe C., Tolley M.K. // CLF Annual Report 66. Oxfordshire, 2004-05.

[9] Neumayer P., Aurand B., Basko M., Ecker B., Gibbon P., Hochhaus D.C., Karmakar A., Kazakov E., Kühl T., Labaune C., Rosmej O., Tauschwitz An., Zielbauer B., Zimmer D. // Phys. Plasmas. 2010. V. 17. P. 103103. dx.doi.org/10.1063/1.3486520

[10] Nersisyan G., Makita M., McKeever K., Dzelzainis T., White S., Nedanovska E., Kettle B., Nicholl R., Williams G., Riley D., Lewis C.L.S. // Phys. Rev. E. 2012. V. 85. P. 056415. doi 10.1103/PhysRevE.85.056415

[11] Arora V., Naik P.A., Chakera J.A., Bagchi S., Tayyab M., Gupta P.D. // AIP Advances. 2014. V. 4. P. 047106. doi 10.1063/1.4870946

[12] Andreev A.A., Limpouch J., Iskakov A.B., Nakano H. // Phys. Rev. E. 2002. V. 65. P. 026403.

doi 10.1103/PhysRevE.65.026403

[13] Theobald W., Akli K., Clarke R., Delettrez J.A., Freeman R.R., Glenzer S., Green J., Gregori G., Heathcote R., Izumi N., King J.A., Koch J.A., Kuba J., Lancaster K., MacKinnon A.J., Key M., Mileham C., Myatt J., Neely D., Sangster T.C. // Phys. Plasmas. 2006. V. 13. P. 043102. doi 10.1063/1.2188912

[14] Griem H.R. Spectral Line Broadening by Plasmas. New York: Academic, 1974.

[15] Виноградов А.В., Собельман И.И., Юков Е.А. // Квантовая электроника. 1974. Т. 1. № 2. С. 268-278.

[16] Рагозин Е. // Квантовая электроника. 1977. В. 4. № 10. C. $2262-2265$.

[17] Evans R.G., Clark E.L., Eagleton R.T., Dunne A.M., Edwards R.D., Garbett W.J., Goldsack T.J., James S., Smith C.C., Thomas B.R., Clarke R., Neely D.J., Rose S.J. // Appl Phys. Lett. 2005. V. 86. P. 191505. doi 10.1063/1.1920422.

[18] Chung H.-K., Chen M.H., Morgan W.L., Ralchenko Y., Lee R.W. // High Energy Density Physics. 2005. V. 1. N 1. P. 3-12. 
[19] Welch D.R., Rose D.V., Oliver B.V., Clark R.E. // Nucl. Instrum. Methods Phys. Res. A. 2001. V. 464. P. 134-139. doi 10.1016/S0168-9002(01)00024-9

[20] Magee N.H., Abdallah J., Colgan J., Hakel P., Kilcrease D.P., Mazevet S., Sherrill M., Fontes C.J., Zhang H.L. // AIP Conf. Proc. 2004. V. 730. P. 168-179.

[21] Держиев В.И., Жидков А.Г., Яковленко С.И. Излучение ионов в неравновесной плотной плазме. М.: Энергоатомиздат, 1986. 43 c; Derzhiev V.I., Zhidkov A.G., Yakovlenko S.I. Radiation of Ions in a Nonequilibrium Dense Plasma. M.: Energoatomizdat, 1986. 43 p.

[22] Teng Z.X., Chen C.Y., Yan S.X., Wang Y.S., Yang F.J., Sun Y.S. // J. Quant. Spectrosc. Radiat. Transfer. 1999. V. 61. N 1. P. $123-129$.

[23] Кондратьев Д.А., Бейгман И.Л. // Краткие сообщения по физике ФИАН. 2010. В. 37. № 27. С. 3-14; Kondrat'ev D.A., Beigman I.L. // Bulletin of the Lebedev Physics Institute. 2010. V. 37. N 12. P. 3-14. doi 10.3103/S1068335610120018

[24] Andiel U., Eidmann K., Witte K. // Phys. Rev. E. 2001. V. 63. P. 026407. doi 10.1103/PhysRevE.63.026407

[25] Djaoui A. A User Guide for the Laser-Plasma Simulation Code: MED103. Chilton, Didcot, Oxfordshire, Rutherford Appleton Laboratory, 1996.

[26] Лисица В. // УФН. 1977. Т. 122. № 3. С. 449-494; Lisitsa V.S. // Sov. Phys. Usp. 1977. V. 20. P. 603-630. doi 10.1070/PU1977v020n07ABEH005446 